\title{
Experience Centred Design for a Robotic Eating Aid
}

\author{
Javier Jiménez Villarreal \\ Mobile Life@ @ICS \\ Kista, Sweden \\ javierjv@sics.se
}

\author{
Sara Ljungblad \\ Mobile Life@ @ICS \\ Kista, Sweden \\ saral@sics.se
}

\begin{abstract}
We discuss how an experience centred approach to robotic design might lead to new design spaces and products that are more engaging and better meet users' needs and lifestyles. To support the statement, we present preliminary data from a long-term user study on an eating aid robot.

\section{Categories and Subject Descriptors}

H.5.2 [Information Interfaces and Presentation]: User Interfaces - theory and methods, user-centred design

\section{General Terms}

Design, Human Factors, Theory.

\section{INTRODUCTION}

The purpose of this paper is to study a robotic eating aid from a experience centred perspective aiming to understand the specific use situation and gain valuable knowledge for the design of future robotic aids. We will discuss how the use of a robotic aid relates to the user's social life in different terms than replacing human assistants. We will also present the importance of taking a holistic perspective of aesthetics. Such perspective views aesthetical qualities as emerging from interaction with an object rather than being inherent in it, and considers them as equally important for successful design as efficiency or usability.
\end{abstract}

\section{EXPERIENCE CENTRED APPROACH TO HRI}

Our approach takes inspiration from the experience centred design field of Human Computer Interaction [4]. Thus, instead of focusing on usability or input or output interaction issues, we strive for taking a holistic and deepened perspective into understanding the users' experiences. For that it is indispensable to take users and not technology or goals as the center of our design methodology. We hope that such a perspective can open-up the design space and teach us about for example specific ethical issues that will emerge preventing us from designing unethical products or products that fail to meet the users needs, and that in the end may not be used.
Copyright is held by the author/owner(s).

HRI'11, March 6-9, 2011, Lausanne, Switzerland. ACM 978-1-4503-0561-7/11/03.

\subsection{What is a disability?}

What does it mean to consider disabilities from a user and experience centred perspective? Overall, having a disability suggests that there is situation where our ability can be improved to achieve a certain goal. It is important to differentiate disabilities from handicaps for designers to better approach users needs. "A handicap results from the interaction between a disability and the environment; it does not flow naturally from the disability alone" [1]. Even people without disabilities can be "handicapped" in certain environments, for example when needing to speak with a microphone to be heard in a big hall [5]. Taking an experience centred perspective on designing for a disability means to for example trying to consider aesthetics, fashion, identities and lifestyles in this situation, thus going beyond functionality and physical characteristics [2].

\subsection{What is a desired assisted eating situation?}

What are the important qualities in an assistive eating experience from a user and experience centred perspective? Hammel, Jacobson and Pirinen [2] conducted a lab study of a robotic assistant with several functions, such as prepare a meal, fetch and carry objects, feeding and more. They found that people's primary reason to want a robot was to gain control and independence in their home and work environment. Our approach is to conduct a long-term study, where people use an eating aid on more or less a daily basis in their home, in order to learn about these and other aspects in more detail.

\section{EXPERIENCE PERSPECTIVES ON AN EATING AID}

Below we will describe the robotic arm Bestic, and discuss important ethical perspectives to consider about robotic eating aids.

\subsection{Bestic}

Bestic supports eating for people who cannot feed themselves due to disabilities in hands and arms. Bestic is designed to look like a kitchen aid, is equipped with a robotic arm that has a spoon attached and is programmed to simplify the action of picking up food from the plate (See Figure $1)$. The user can steer the product with different operating devices adapted to their disability. The user decides which area of the plate should collect the food from by steering the spoon and

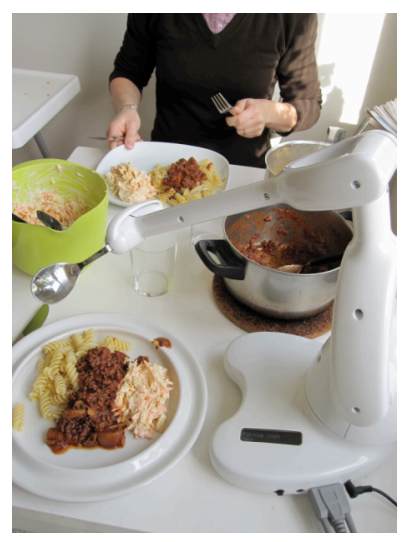

Figure 1. About to eat lunch, using Bestic. 
presses a button to automatically collect the food from that area. Normally, someone else has to chop the food, serve it and collect pieces that may accidentally be pushed outside the plate. Bestic cannot support any other user activities, and is thus only intended to support the act of feeding oneself.

We are currently conducting long-term studies on two people that are using Bestic as a tool in their everyday. The interviews are held in the users' homes as semi structured conversations about their experiences of Bestic. Below we will present some early examples of our data and discuss some ethics concerns as well as users' experiences.

\subsection{Social isolation or freedom of choice?}

One overall ethical concern that has been raised with this kind of product is the social impact that it's introduction might have. For example, social isolation is seen by some as the logical result of lowering assistants or familiars workload in regards to help disabled people. Sharkey [6] for example argues that even if such robots may support independence, users may end up being left "in the exclusive care of machines". Sparrow and Sparrow [8] argue, "regardless of the intentions of the designers and manufacturers, in reality robots will inevitably be used to replace human staff".

We believe that these concerns have their roots in the view of robots as designed mainly to perform tasks to alleviate human workload. In contrast, designing from user-centered approaches focusing on improving users' experience might lead to products that enhance different social aspects. We argue that a robotic product can support a very specific need, where a personal assistant is not necessarily part of the desired experience, thus addressing successfully the need and user's preferences without eliminating the need for an assistant.

Karl - one of Bestic users who is 35 years and is usually fed by an assistant - explains his experience:

"It is directly more pleasant to eat yourself than to have an assistant. His partner describes that: - It's much more private, you can talk about whatever you want. Karl about assistants: - They are a bit uncomfortable when they give me food. I'm thinking of what may cause that. It's an intimate situation."

This shows how in fact this user may simply want to eat and not necessarily be social with the person who is assisting eating. Using a robotic product such as Bestic however, may allow for intimate and private conversations while having a meal with someone. Thus, from a user and experience centred perspective it is important to understand what the users different lifestyles are, their specific needs, interests and which kind of solution that they would prefer and why.

\subsection{Aesthetics and acceptance}

Assistive design including assistive robotics usually focus on functionality rather than aesthetical qualities or users' desires and lifestyles. To Hammel, Jacobson and Pirinen [2] "this may lead to products and environments that are unattractive and even stigmatizing to users". This suggests the importance of considering aesthetics in the design of robotic aids.

Aesthetics includes the qualities perceived by all our sensory elements [7], when striving for a holistic perspective of the experience. Here, it is important to consider them -and their desired effects- during the design process in coherence with the design goals [3]. From an experience centred perspective our goal is thus not only to create products that are only efficient and easy to use, but also pleasing, enjoyable or seductive. From our longterm study we are learning about how users with different disabilities and life situations affects the experience of the artifact. For example, we are learning about the importance of a smooth movement of the spoon, the need to manage a variety of foods such as spaghetti, soup and ice-cream, and that it is more fun to eat without spilling food.

What are the robotic properties that most efficiently support feeding oneself? Perry et al. [5] stress that there is a risk of confusing the task with a particular way of performing it, when designing for people with disabilities. Even if today's solution is to be fed by an assistant, designing something that is reminiscent to an assistant's way of feeding may not lead to a desired assisted eating experience. Similarly, copying people without disabilities and physically moving the users arm may not necessarily improve the experience of feeding oneself. Thus, when designing any robotic aid with technical properties such as autonomy, the experience is an important ethical consideration.

\section{CONCLUSION}

Designing for robotic eating aids raise very specific design concerns, including ethics and aesthetics that relates to the users experience. We have discussed very specific ethical perspectives that relates to social isolation and acceptance as shaped from aesthetics. Based on our preliminarily results from a long term study, we are striving to increase our understanding of what it means to design successful robotic eating aids - from the users perspective and their everyday experience.

\section{ACKNOWLEDGMENTS}

Thanks to our Bestic users for valuable insights. This work is part of the LIREC project, in the European Community's Seventh Framework Programme (FP7/2007-2013) under grant agreement $\mathrm{n}^{\circ} 215554$

\section{REFERENCES}

[1] R. Amundsson, 1992. Disability, handicap, and the environment. Journal of Social Philosphy Vol 3 (1), p105-119.

[2] Susanne Jacobson and Antti Pirinen. 2007. Disabled persons as lead users in the domestic environment. In Proceedings of the 2007 conference on Designing pleasurable products and interfaces (DPPI '07). ACM, New York, NY, USA, 158-167.

[3] S. Lundgren. Teaching and Learning Aesthetics of Interaction. Doctoral thesis, Chalmers University of Technology, 2010.

[4] J. McCarthy and P. Wright. 2004. Technology as experience. Interactions 11, 5 (September 2004), 42-43.

[5] P. Perry. Macken E. Scott, N Janice L. McKinley 1997 Disability Inability and Cyberspace. In Eds. Friedman. Human Values and the design of computer technology.Cambridge, CSLI Publications.

[6] N. Sharkey 2008. The ethical frontiers of robotics Vol. 322. no. 5909, pp. 1800 - 1801

[7] R. Shusterman. Somaesthetics: A Disciplinary Proposal. The Journal of Aesthetics and Art Criticism, 57(3):299, 1999

[8] R. Sparrow, and L. Sparrow. "In the hands of machines? The future of aged care." Minds and Machines 16, no. 2 (August 2006): 141161 
